\title{
Commentary-based Video Categorization and Concept Discovery
}

\author{
Janice Kwan-Wai Leung
}

\begin{abstract}
Social network contents are not limited to text but also multimedia. Dailymotion, YouTube, and MySpace are examples of successful sites which allow users to share videos among themselves. Due to the huge amount of videos, grouping videos with similar contents together can help users to search videos more efficiently. Unlike the traditional approach to group videos into some predefined categories, we propose a novel comment-based matrix factorization technique to categorize videos and generate concept words to facilitate searching and indexing. Since the categorization is learnt from users feedback, it can accurately represent the user sentiment on the videos. Experiments conducted by using empirical data collected from YouTube shows the effectiveness of our proposed methodologies.
\end{abstract}

\section{Introduction}

Starting from last decade, World Wide Web is developed into the second generation Web 2.0. Thanks to this newest development, people can control over the data in the Internet instead of just retrieve data. Furthermore, it led to the evolution of web-based communities. Social networks such as forums, blogs, video sharing sites are examples of applications.

Recent years online video sharing systems are burgeoning. In video sharing sites, users are allowed to upload and share videos with other users. YouTube is one of the most successful and fast-growing systems. In YouTube, users can share their videos in various categories. Among these video categories, music is the most popular one and the number of music videos overly excess that of other categories $[1,7]$. Users are not only allowed to upload videos but tag videos and leave comments on them as well. With more than 65,000 new videos being uploaded every day and 100 million video views daily, YouTube becomes a representative community among video sharing sites.

Due to the incredible growth of video sharing sites, video searching is no longer a easy task and more effort should be paid by users to search their desire videos from the entire video collection. To address this problem, grouping videos with similar contents together is necessary. Currently, videos on YouTube are only coarsely grouped into some predefined high level categories (e.g. music, entertainment, sports, etc). Videos in a single category still span through a wide range of varieties. For example, in the music category, we may find music from various countries or with different musical styles. Though some other video sharing sites, such as DailyMotion and MySpace, have a lower level of category for music videos, the categorizes just follow the basic music genre. However, people interests on music are not limited to these simple genre. Furthermore, the predefined categories maybe too subjective to capture the real interest of the majority of users since they are only defined by a small group of people. Finally, the current categories on YouTube are fixed and it is hard to add/remove categories too often. As time goes by, some categories may become obsolete and some new topics may be missing from the categories.

These observations motivate us to explore a new way of video categorization. In this work, we propose a novel comment-based clustering technique by utilizing user comments for achieving this goal. Unlike the traditional approaches of predefining some categories by human, our categorization is learnt from the user comment. The advantage of our proposed approach is three-fold. First, our approach can capture user interests more accurately and fairly than that of the predefined categories approach. The reason is that we have taken the user opinions into consideration. In other words, the resulting categories are contributed by public users rather than a small group of people; Second, since user interest can be changed from time to time, the categories of our method can be changed dynamically according to the recent comments by users; Finally, as users comments are in the form of natural language, users can describe their opinions in detailed. Therefore, by comment-based clustering, we can obtain clusters which represent fine-grained level ideas.

In the literature, various clustering techniques have been proposed for video categorization [5, 12]. However, this type of techniques did not take user opinion into consideration and thus the clustering results do not capture user interests. On the other hand, researchers have proposed to use the user tags on videos for clustering $[6,4]$. Though 
user tags can somehow reflect user feelings on videos, tags are, in many cases, too brief to represent the complex ideas of users and thus the resulting clusters may only carry highlevel concepts. Another stream of works which use commonly fetched objects of users for clustering [2] also suffer similar shortcomings as tag-based clustering. In [11], they proposed to adopt a multi-modal approach for video categorization. However, their work required lots of human efforts to first identified different categories from a large amount of videos.

We want to remark that although comment-based clustering can theoretically obtain more fine-grained level clusters, it is much more technically challenging than that of tagbased clustering. The reason is that user comments are usually in the form of natural language and thus pre-processing is necessary for us to clean up the noisy data before using them for clustering.

The rest of the paper is organized as follows. Section 2 discusses previous works in the context of social network mining. Section 3 explains our proposed approach for video categorization in video sharing sites. Section 4 briefly introduces our web crawler. Section 5 presents the details of pre-processing of the raw data grabbed by our crawler. Section 6 describes our video clustering algorithm. Section 7 presents and discusses our experimental results. Section 8 concludes the paper.

\section{Related Works}

Since the late eighties, data mining has became a hot research field. Due to the advancing development of technologies, there is an increasing number of applications involving large amount of multimedia. For this reason, researches in the field of data mining are not limited to text mining but multimedia mining. Qsmar R. Zaine et al. [12] developed a multimedia data mining system prototype, MulitMediaMiner, for analyzing multimedia data. They proposed modules to classify and cluster images and videos based on the multimedia features, Internet domain of pares referencing the image of video, and HTML tags in the web pages. The multimedia features used include size of image or videos, width and height of frams, date on which the image or video was created, etc. S. Kotsiantis et al. [5] presented a work to discover relationships between multimedia objects based on the features of a multimedia document. In their work, features of videos such as color or grayscale histograms, pixel information, are used for mining the content of videos.

Motivated by the bloom of social networks, plenty of works have been done involving the study or analysis of online social networks. Different approaches are proposed to discover user interests and communities in social networks. Tag-based approach is one of the invented methods. In [6],
Xin Li et al. developed a system to found common user interests, and clustered users and their saved URLs by different interest topics. They used the dataset from a URLs bookmarking and sharing site, delicio.us. User interests discovery, and user and URLs clustering were done by using the tags users used to annotate the content of URLs. Another approach introduced to study user interests is usercentric which detects user interests based on the social connection among users. M. F. Schwartz et al. [8] discover people's interests and expertise by analyzing the social connections between people. A system, Vizster [3], was designed and developed to visualize online social networks. The job of clustering networks into communities was included in the system. For this task, Jeffrey and Danah identified group structures based on linkage. Except the use of sole tag-based or user-centric approaches, there are works done with a hybrid approach by combing the two methods. In [4], user interests in del.icio.us are modeled using the hybrid approach. Users are able to make friends with other users to form social ties in the URLs sharing network. Julia Stoyanovich et al. examined user interests by utilizing both the social ties and tags users used to annotate content of URLs. Some researchers proposed the object-centric approach for social interests detection. In this approach, user interests are determined with the analysis of commonly fetched objects in social communities. Figuring out common interests is also a useful task in peer-to-peer networks since shared interests facilitate the content locating of desire objects. Guo et al. [2] and K. Sripanidkulchai [9] presented in their works the algorithms of examining shared interests based on the common objects users requested and fetched in peer-to-peer systems.

\section{Interest-based Video Categorization for Video Searching}

With the ceaseless growth of media content, it is increasingly a tense problem for video searching. It is usual that users hardly find their desire videos from the immense amount of videos. There are two main directions to ease the process of video searching, one is enhancing the text-based search engine whilst the other one is designing a better directory. In this paper, we focus on the former approach.

Though many video sharing sites allowed tagging function for users to use tag to annotate videos during the upload process, it is very common for user to tag videos by some high level wordings. As such, tags are usually too brief for other users to locate the videos by using the text-based search engine. In our method, as user comments usually describe the video in details, we can use them for video clustering to obtain fine-grained categories. By identifying the concept words for each categories, we can use them as latent tags for the corresponding categories in order to facil- 
itate the video searching process.

In music domain, music videos in sharing systems are always categorized according to their types of musical sounds (e.g. pop, metal, country, etc.) under the music genre. However, except music styles, people may have many different attitudes and preferences (e.g. appearance of singers, event of performance, age of songs, etc) towards music in different regions. Therefore, to categorize music based on people interests, music genre is not a good categorical construct for video searching.

Our aim is to find a categorization where videos in each video group are representing a popular topic of interest. In our algorithm, interests are modeled by clustering videos into groups with the utilization of user-left comments. Previously, computer scientists have tried many ways to find user interests. Tags are very popular to help in this context [6]. However, two disadvantages would be raised in this manner. First, tags on a video are manually given by the one who uploads the video, thus the tags are just expressing a single user's feeling about the video. Therefore, tags on a video would have a strong bias and are not fair enough to exactly describe what the video is actually about. Furthermore, single-user given tags are definitely not representative of public feelings about the video. Second, videos are often tagged with a small number of words. As such, often fails to give enough description on the video. Therefore, tags are insufficient to provide detailed information about videos. Since comments can be given by any users on any videos as feedbacks, they express different users thoughts about a video. Thus, containing more in-depth information about the videos. Also, allowing every user to leave feedbacks, the number of comments on a video are usually much more than that of tags. Hence, utilizing comments to find out interests instead of tags can solve the above difficulties.

Beside tag-based, some researchers proposed the content-based approach to categorize videos. Using video content as categorizing materials can group similar videos together according to their actual content. Nevertheless, video content itself only provide information about the videos but nothing about users' idea. Consequently, this approach fails to group videos according to user interests. In contrast, user-left comments include users' view about the videos. Therefore, comments can, undoubtedly, be used to categorize videos based on popular user interests.

Video features can also be used to achieve the goal of cluster videos [5]. Video features, however, are hard to be extracted automatically. Due to the limitation of human resources, automatic information retrieval from mass amount of data is preferred. Also, using video features to cluster videos suffers the same shortcomings of content-based as well. Because of information retrieval dealing with text is much easier than video features extraction, and comments, in addition to video content, provide users' views on videos, user-left comments are significant for clustering videos.

\section{Data set collection}

YouTube is a video sharing platform which users can upload their own videos for sharing purpose. Along with each video, a short description can be entered by the uploading user and also comments can be given by other registered users. In this paper, we focused on the user comments of music videos of Hong Kong singers in YouTube.

We first defined a set of 102 Hong Kong singer/group names. Given the set of singer/group names, we developed a crawler to firstly visit the YouTube web site and automatically searches from the site the related videos based on video titles and video descriptions. From the resulting videos, the crawler saves the URL of each videos for further process. For the convenience of gathering user comments, the crawler transforms the fetched URLs to links which link to the pages of "all comments" mode of corresponding videos. With all the transformed video URLs, for each link, the crawler is able to scrape the video web page and grab the video title, all the user comments and user name of who left comments on the video.

In the data set acquired by our crawler, there are 19305 videos, 102 singers and 7271 users in total.

\section{Data Pre-processing}

To ease the process of video searching by discovering the user interest and categorizing videos, larger amount of data is required from video sharing sites. However, just the large-sized collection of text-formatted raw data is not applicable for further processing. Large-sized dataset always need to undergo data pre-processing in the field of data mining. Here is no exception in our algorithm. After crawling YouTube, the mass data need to be pre-processed before performing video clustering.

Here are two steps of data pre-processing involved in our introduced algorithm,

1) Data Cleaning

2) Text Matrix Generation

\subsection{Data Cleaning}

As the comments left on YouTube videos are written in natural languages which consist lots of non-informative words, such as "thank", "you", etc, text processing with such materials must be caution. To avoid resulting a poor clustering,data cleaning is necessary for handling the noisy words.

In natural languages, there are many words that are not informative for clustering. These words would make the 
entire dataset very noisy. Applying a stoplist is one of the ways to clean up these words. Since some words are obviously not informative, it is easy to define a stoplist of noise. With a predefined stoplist non-informative or distractive words can be strained from the dataset. After removing all the useless words by the stoplist, the dataset is then passed to the process of matrix generation.

\subsection{Text Matrix Generation}

Text-formatted data is not easy for further processing, it is more convenient to transform the data from text to matrix representation beforehand.

For example, the dataset can be represented by matrix $A$ of size $n \times m$ where $n$ is number of videos in the dataset and $m$ equals to number of unique case-insensitive words in the dataset. In $A$, each row is a vector of video words and element $a_{i, j}$ is the frequency count of word $j$ occurs in comments left on video $i$.

A dictionary is firstly built with the case-insensitive words in all the comments in the dataset. As comments are all in texts, linguistically, there exist many meaningless words in comments. These meaningless words, e.g. "is", "am", "the", "a", always occur in an extremely high frequency. Therefore, words occur in frequency exceeding a threshold should be discarded. On the other hand, words that seldom occur are probably not important, so words with few occurrence should also be neglected. Therefore, we set an upper bound and a lower bound for word occurring frequency. All the words with frequency less than the lower bound or larger than the upper bound are filtered out. After filtering all the meaningless words, dictionary can then be built and matrix can be generated as well.

\section{Popular Interests Discovery and Video Cat- egorization via Clustering}

In order to facilitate the video searching process, constructing a video category based on the user interests is crucial.

With the reason that users' interests are reflected from the comments they left on videos, grouping similarly commented videos together is a possible way to provide a good video categorization. Since the objective of clustering is to distinguish substantial amount of data and group similar objects together, clustering is an adequate algorithm for constructing a video category that can guide user to his/her desire videos.

Figure 1 shows the procedures of discovering popular interests and categorizing Hong Kong singer videos from YouTube.

\subsection{Video Clustering}

For our purpose of building a good video category for easier video searching, Non-negative Matrix Factorization (NMF) is the chosen clustering algorithm [10]. We propose to apply NMF for clustering based on three reasons. First of all, NMF is a bi-clustering method. With a bi-clustering algorithm, comment words and videos can be clustered simultaneously. Thus, the main characteristics of video groups can be drawn while grouping videos with similar user views together. Additionally, NMF does not provide an absolute assignment of videos to groups. Absolute assignment clustering algorithms are not suitable for singer video clustering. In practice, a video can belongs to multiple groups. For example, a classic music video can be performed by a singer who is passed away. The video is said to be in both "classic" group and "died singer" group. As NMF calculates possibility coefficients of each video to different groups, a single video can be assigned videos to multiple groups. Finally, NMF is effective for clustering. Since we need to cluster a large amount of data, effectiveness is one of the concerns. An effective low-dimensional linear factor model is desired.

Comments on a video often capture users feelings about the video or describe the video. Videos are clustered into the same group if they bear comments with similar contents. Similar videos, therefore, can be grouped together and with their characteristics be revealed as popular user-interests.

Let $A$ be the $n \times m$ video-word matrix generated in the process of data pre-processing, where $n$ and $m$ are the number videos and number of words in dictionary respectively. As all the elements in $A$ are the occurrence counts of words in documents, they are greater or equal to zero. This makes matrix $A$ a non-negative matrix.

Since the importance of a term to a document can be reflected by it's number of appearance, the well-known keyword measure in Information Retrieval $t f-i d f$ is adopted for extracting important words. Within the dataset, all the comments of a video is aggregated and considered as a document. Importance of term $i$ in document $j$ is $w_{i, j}$ which is computed by using $t f_{i, j}$ (term frequency of term $i$ in document $j$ ) and $i d f_{i}$ (inverse document frequency of term $i$ ). Terms that are important to a document are expected to appear many times in the document. For this reason, the term frequency is used to measure the normalized frequency of a term in a document. Suppose there are $t$ distinct terms in document $j, t f_{i, j}$ can be computed as,

$$
t f_{i, j}=\frac{f_{i, j}}{\sqrt{\sum_{k=1}^{t} f_{k, j}^{2}}}
$$

where $f_{i, j}$ is the number of times that term $i$ appears in document $j$. As words appear in many documents are not useful for distinguishing documents, a measure $i d f$ is used 


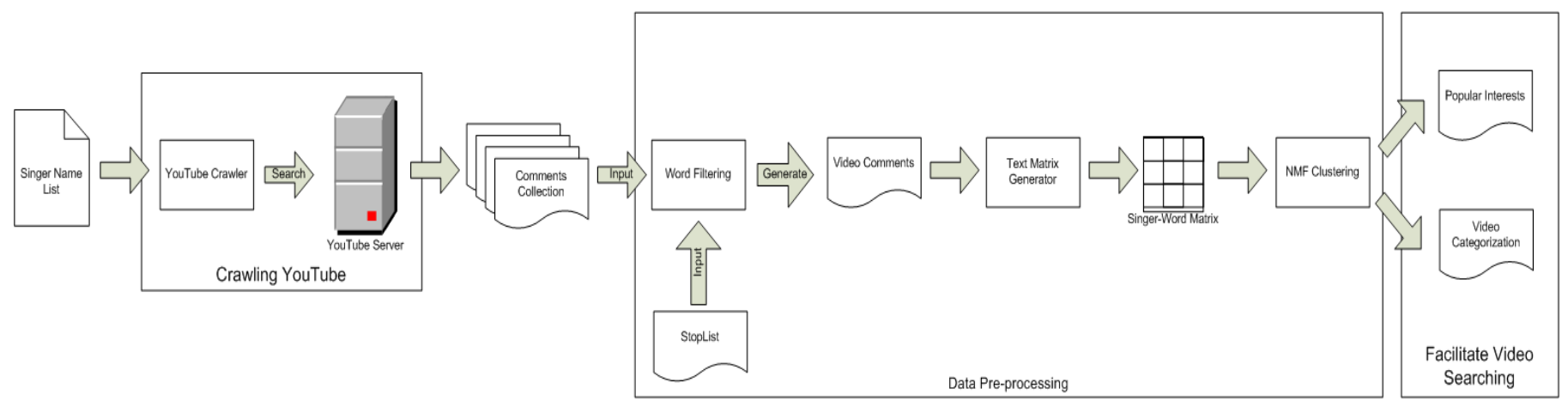

Figure 1. Popular interests discovery and video categorization of Hong Kong singer videos in YouTube

to scale down the importance of these widely-used terms. The inverse document frequency of term $i$ is defined as,

$$
i d f_{i}=\log \frac{N}{n_{i}}
$$

where $N$ is the total number documents in the dataset, and $n_{i}$ is number of documents that containing term $i$.

After computing the term frequency and inverse document frequency, the importance weight of a term $i$ in document $j$ is defined as the combination of $t f_{i, j}$ and $i d f_{i}$,

$$
w_{i, j}=t f_{i, j} \times i d f_{i}
$$

The greater the weighting, the more the important is the term to the respecting document.

From matrix $A$, a non-negative matrix $X$ can be produced by calculating the importance weights. Each element in $X$ is defined as,

$$
x_{i, j}=w_{i, j}=\frac{a_{i, j}}{\sqrt{\sum_{k=1}^{t} a_{k, j}^{2}}} \times \log \frac{N}{n_{i}}
$$

By fitting a k-factor model to matrix $X$, where $k$ equals to number of groups to be obtained, $X$ is decomposed to two non-negative matrices $W$ and $H$, such that $X=W H+$ $U$. After matrix decomposition, $W$ is in size of $n \times k$ and $H$ is in size of $k \times m$.

Our objective is to find $\mathrm{W}$ and $\mathrm{H}$ such that $X \approx W H$. By iteratively updating $W$ and $H$, we can obtain $W$ and $H$ by minimizing the following function,

$$
F(W, H)=\|X-W H\|^{2}
$$

with respect to $W$ and $H$ and subject to constraints that $W, H \geq 0$.

Figure 2 shows the decomposition of video dataset matrix. From the resulting matrices, relationships between words videos and clusters are revealed. Matrix $W$ shows the relationships between videos and different clusters, whilst $H$ clarifies the relationships between words and clusters. In $W$, value held in $w_{n, k}$ is the coefficient indicated how likely video $n$ belongs to cluster $k$. To fit the purpose of our research, we have refined the method of group assigning in NMF. The original NMF algorithm assigns an object to a group in a maximum coefficient approach. However, in our method, a video $n$ is treated to be in group $k$ if $w_{n, k}$ has the a value greater than a threshold $\beta_{k}$ within vector $n$ in $W$, where the value of threshold $\beta_{k}$ is data dependent. The threshold should be chosen in a coefficient distribution depend manner. Videos can then be grouped into clusters based on their similarities. We define the set of cluster for video $V_{n}$ that it belongs to as,

$$
C_{n}=\left\{k \in K \mid \forall W_{n, k}>\beta_{k}\right\}
$$

where $K$ is set of all clusters.

Matrix $H$ provides the information about the characteristics of the video groups. Concept words of a cluster can be found with $H$ as $h_{k, m}$ is the coefficient of the term $m$ belongs to cluster $k$. For each cluster, the top 10 words, with respect to the term-cluster coefficient, are considered to be the concept words for the cluster. Hence, stating the properties of a group of videos.

\section{Experimental Evaluation}

A proof-of-concept experiment was done to study the Hong Kong social culture in regional music domain. An Intel(R) Core(TM)2 Quad 2.40GHz PC with 4GB RAM was used to conduct our experiment. Our web crawler was implemented in $\mathrm{VC}++$ and the core algorithm was implemented in Matlab. 


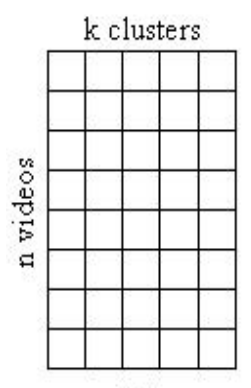

W

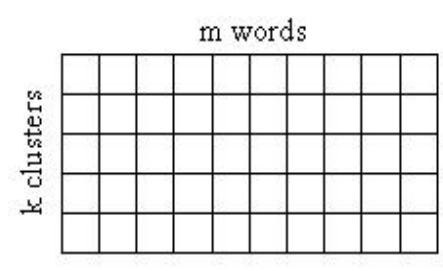

$\mathrm{H}$
Figure 2. NMF decomposition for video clustering.

\subsection{Empirical Setting}

As the videos were grabbed by searching from the YouTube site with predefined list of singer names, there are possibilities that some videos are grabbed more than one time. For those videos preformed by more than one singer, as long as there are more than one singer names annotated in the video title, the video will be collected in times equals to the number of hits the predefined singer name hits the video title. To achieve a more accurate clustering result, duplicated videos are removed form the dataset.

In comments, users are used to mention the singer names when they are commenting on him/her. This will make the singer names dominate in every group of concept words. However, it is not conspicuous enough to reveal interests by singers. Therefore, in our experiment, we add singer names to the stoplist as well.

Furthermore, some videos are less popular or just been uploaded for a short time that only have a few comments. These videos which have relatively few words are informativeless for video clustering. Videos which have less than 30 valid words are eliminated.

The videos are clustered into $k$ groups with the clustering algorithm discussed in section 7, where $k$ is experimentally set as 20 . The experiment was done twice, once with threshold $\beta_{i}$ regarding cluster $i$ to be mean coefficient of all videos,

$$
\beta_{i}=\text { meanCoef }_{i}=\frac{\sum_{j=1}^{n} w_{j, i}}{n}
$$

To compensate the poor performance caused by the extremely uneven distribution of coefficient, we chose the threshold to be mean coefficient plus standard deviation of all videos for the second experiment. $\beta_{i}$ regarding cluster $i$ is defined as,

\begin{tabular}{|c|l|}
\hline Group & Concept Words \\
\hline 1 & beautiful lyrics melody \\
\hline 2 & female makeup dress \\
\hline 3 & cute pretty handsome \\
\hline 4 & sex photos scandal \\
\hline 5 & funny hilarious laughing \\
\hline 6 & rap raps hip \\
\hline 7 & movie film story \\
\hline 8 & cantonese mandarin language \\
\hline 9 & commercial pepsi coke \\
\hline 10 & piano piece ear grade \\
\hline 11 & japanese japan korean \\
\hline 12 & china olympic games \\
\hline 13 & old classic memories \\
\hline 14 & dance dancer moves \\
\hline 15 & guitar band rock \\
\hline 16 & award tvb gold \\
\hline 17 & english chinese accent \\
\hline 18 & sad legend died \\
\hline 19 & together couple two \\
\hline 20 & voice pretty talent \\
\hline
\end{tabular}

Table 1. Latent video categories discovered in Hong Kong music video domain from YouTube

$\beta_{i}=$ meanSdCoef $_{i}=\frac{\sum_{j=1}^{n} w_{j, i}}{n}+\sqrt{\frac{1}{n} \sum_{j=1}^{n}\left(w_{j, i}-\frac{\sum_{j=1}^{n} w_{j, i}}{n}\right)^{2}}$

where $n$ is total number of videos being clustered.

\subsection{Video Categorization}

Since video clustering is a complete clustering analysis, music categories and trends in Hong Kong can be found by clustering the videos. We deployed NMF as our clustering method. Applied the clustering algorithm to the video dataset in the way discussed in Section 5.2, with the experimentally chosen number of cluster of 18 , videos were clustered into groups based on the words in their comments. The mean coefficient of videos to a cluster is set as the critical point. Videos with coefficient higher than the critical point of a cluster are said to be in that cluster. Under this strategy, videos can belong to several clusters as they may have multiple characteristics. Table 1 shows the discovered categories from our dataset.

Unlike the generic music video categorization of some famous video sharing sites, such as DailyMotion divides music videos into eight classes (Pop, Rock, Rap, R\&B, 


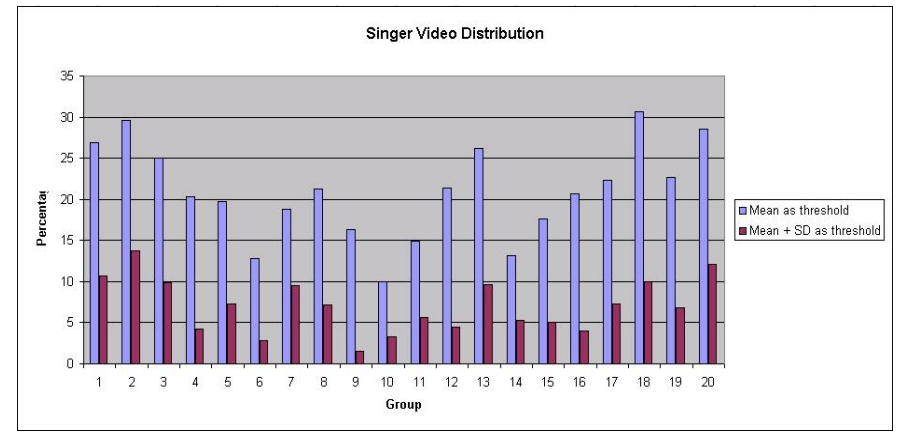

Figure 3. Singer video distribution in YouTube.

\begin{tabular}{|c|l|c|c|}
\hline & & \multicolumn{2}{|c|}{ Precision } \\
\hline Group & Concept Words & $\begin{array}{l}\text { Mean as } \\
\text { Threshold }\end{array}$ & $\begin{array}{l}\text { Mean + SD } \\
\text { as Thresh- } \\
\text { old }\end{array}$ \\
\hline A & sex photos scandal & $21.64 \%$ & $81.58 \%$ \\
\hline B & old classic memories & $61.04 \%$ & $78.16 \%$ \\
\hline C & sad legend died & $35.86 \%$ & $60.34 \%$ \\
\hline D & together couple two & $64.44 \%$ & $79.82 \%$ \\
\hline \multicolumn{2}{|c|}{ Average } & $45.75 \%$ & $74.96 \%$ \\
\hline
\end{tabular}

Table 2. Precision of objective clusters

Jazz, Metal, Covers, and Electros), we categorized videos of local singers into twenty classes which are far more specific.

From our clustering result, we noticed that videos of singers are not only limited to general music videos, but also funny clips, award presentations, commercial advertisements as well as event promotion clips. Looking at the music videos alone, by clustering users' comments, we found that people's attitude towards Hong Kong music are not only target on the music styles. There are also other features of music people are interested at, like languages, age of music, music instruments, type of singers, singer's voice, composition goodness, etc.

Furthermore, categorize singer videos with the proposed clustering algorithm, people can identify dance-oriented videos (Group 14), cross-culture produced music (Group 11) or even movie theme songs (Group 7) easily. Other than simply categorizing singer video clips, some up-todate news in the local music circle, like scandals (Group 4), can also be found.

Figure 3 illustrates the distribution of Hong Kong singer videos in YouTube according to the proposed algorithm using mean and mean $+\mathrm{sd}$ as thresholds. From the figure, we can see that the distribution of videos diverse over different threshold values. With the mean coefficient as the threshold, compared to the video groups resulted from the algorithm with mean + SD coefficient as threshold, larger groups of videos can be obtained. In the other words, algorithm associated with a smaller group assigning threshold would result heavier overlapped video groups.

The video clustering results are evaluated by human experts. To make the evaluation less controvertible, we only show the precisions of objective video groups in Table 2 where groups A, B, C, D are cluster 4, 13, 18, 19 in our clustering. In the table, we noticed that assigning videos to groups with a smaller threshold may sometimes lower the precision. This will be caused in the groups which are very distinct to others. As a video group is too specific, the video-group coefficients to the group hold the extreme values. Also, closely related videos to the distinct group is always much fewer than videos which do not. Hence, videos are condense at the lower extreme side. As a result, lowered the mean coefficient and caused the poor precision. On the other hand, we can see that the algorithm which assigns videos into groups with a larger threshold yields far better precisions. The average precision of the larger-threshold clustering groups in the table is $74.96 \%$ whilst that of the lower-threshold clustering is just $45.75 \%$. The difference between the precisions resulted from clustering with the two different thresholds reflects the degree of extraordinary of the video group. The larger the difference, the more the special the group is. For example, in group 4, the two precisions differ from each other by a large percentage at about $60 \%$, and from the concept words we can know that this group is about scandal of singers involving their sex photos. This is obviously an extremely distinct group.

\subsection{User Comments vs User Tags}

As tags are believed to be an accurate description of an object and have been widely used for finding user interests and grouping objects, it is necessary to examine the virtues of user comments over tags before utilizing comments to capture popular interests and categorize videos to facilitate the video search in video sharing sites. One important observation from our experimental results is that user comments usually contains more in-depth information than that of user tags. Table 3 shows both the top 10 concept words found from user comments and the top 10 user tags of four clustered groups. From the concept words in the user comments, we can make a reasonable prediction that cluster I is about some music videos of some old songs. From the user tags, however, we can only find some singer names or some high-level descriptions (e.g. music, mv, mtv). Same as cluster II, from the concept words, this cluster is probably talking about some superstars who are already died. Nevertheless, the most frequent tags are only names of those dead superstars which do not reveal the low-level description of the group. Cluster III is the similar case as the above 


\begin{tabular}{|c|c|c|}
\hline \multirow{2}{*}{ Cluster I } & Top 10 concept words in user comments & old classic memories drama childhood love 80 s memory loved san \\
\hline & Top 10 frequent user tags & chinese chan mv cheung wong love music mtv top anita \\
\hline \multirow{2}{*}{ Cluster II } & Top 10 concept words in user comments & sad legend two died missed heaven star superstar crying talented \\
\hline & Top 10 frequent user tags & cheung chan leslie anita mui chinese mv danny hong wong \\
\hline \multirow{2}{*}{ Cluster III } & Top 10 concept words in user comments & guitar solo band rock cover drummer chords intro crap violin \\
\hline & Top 10 frequent user tags & chinese beyond wong kong cheung ka kui hong nicholas paul \\
\hline \multirow{2}{*}{ Cluster IV } & Top 10 concept words in user comments & sex photos stupid fake victims private innocent scandal girls stop \\
\hline & Top 10 frequent user tags & gillian chung sex photo edison chen gill cheung cecilia chan \\
\hline
\end{tabular}

Table 3. Examples of user comments and user tags in four video clusters

\begin{tabular}{|c|c|c|c|c|c|}
\hline & \multicolumn{4}{|c|}{ Concept Words from Group } \\
\hline & & A & $\mathrm{B}$ & $\mathrm{C}$ & $\mathrm{D}$ \\
\hline \multirow{4}{*}{ Percentage of Videos from Group } & A & $34.04 \%$ & $4.02 \%$ & $0 \%$ & $2.13 \%$ \\
\hline & $\mathrm{B}$ & $0 \%$ & $15.79 \%$ & $0 \%$ & $0 \%$ \\
\hline & $\mathrm{C}$ & $0.84 \%$ & $0.84 \%$ & $7.58 \%$ & $0.84 \%$ \\
\hline & $\mathrm{D}$ & $0 \%$ & $10.17 \%$ & $3.39 \%$ & $5.26 \%$ \\
\hline
\end{tabular}

Table 4. Percentage of videos with tags covering concept words across groups

two clusters. Concept words from user comments state that this group is about the band sound and rock music but the tags only list out the name of a local popular band, "Beyond", and some of the band members. Tags of the other clusters suffer the similar problem as the above mentioned clusters. From the table, we can see that the user tags actually agree with our discovered concept words though the tags just exhibit the high-level sketch of the groups. In the other words, our algorithm gives an in-depth characterization of the videos with the concept words which the characterization cannot be exposed by the user tags, and in the mean time, the concept words achieve a strong agreement with the tags.

From this observation, we can conclude that if we want to obtain clustering results in a more fine-grained level, using comment-based clustering technique is more suitable. For the purpose of social culture discovery, it is beyond doubt that fine-grained level clustering result is more desirable.

To give a more in-depth analysis of comments and tags, we have compared concept words against tags in different clusters. Table 4 record the portion of videos whose tags cover the concept words of different groups and there are two major observations from the table. First, we can see that there are at least $65 \%$ of videos whose tags cannot cover the concept words of the group they belongs to. This implies tag-based clustering cannot completely capture user opinions and video content. Second, we can see that the concept words of each group are mostly covered by tags of its own group. This once again verify the accuracy of our proposed method.

\subsection{Relationship Discovery via Clustering}

Apart from video categorizing and indexing, we can also discover the relationships between singers with the video comments. Music videos always include singers in it. When users leaving comments on a video, the content of the comment is not limited to the video itself but also the singers. Within the comments, some talk about properties of singers, such as styles, the active ages, appearance, etc. Practically, except characteristics, users also tend to talk and discuss the recent hot news about singers. As such, in addition to the videos, user-left comments also describe the singers. In this context, singer relationships can be found from video comments.

To reveal the relationships between singers, singers should be clustered into groups based on their similarities and relatedness. To this end, the set of comments should be reorganized to a form that suits the purpose of singer clustering. In this context, comments are no longer grouped by videos but by singers according to the singers presentence in the videos. Each singer has his/her collection of comments. The adopted clustering algorithm tends to group singers with similar descriptive words together. As such, with all the descriptions and hot news about singers, singers can be grouped based on their style, characteristics or special relationships. From the clustering result, not only the common properties of singers can be found, but also their relationships like members of a band sound, couple, etc.

Applying the same clustering algorithm introduced before with matrix $X$ in the algorithm set to be the singerword matrix, singers can be clustered into groups. Here 


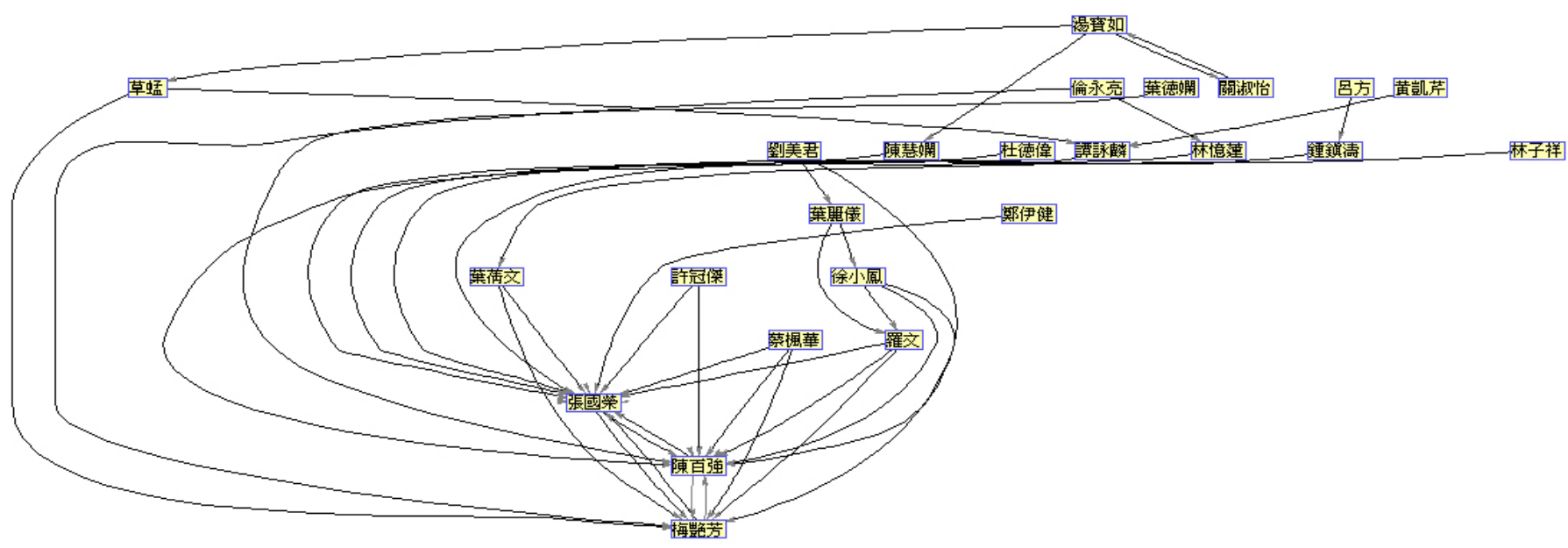

Figure 4. Singer Relationship.

we visualize the relationships between singers in a singer network based on their similarities reflected from the comments. With a visualized singer network, not only the relationships between singers can be shown but also the hidden phenomenon. Due to the paper size, Figure 4 illustrate only partial of the singer network. In this graph, the singers are relatively closely connected to each others than to other singers and thus form a subnetwork. In this subnetwork, all the nodes are active singers in 80 s, this is one of their relationships. The network is a directed graph which the singer being pointed to is highly referenced by the singer who points to him/her. Grasshopper, at the top left corner is pointing to Anita Mui at the bottom as the members of Grasshopper are students of Anita Mui. Leslie Cheung, Danny Chan and Anita Mui are famous singers and many other singers have used their songs for performance. This can be reflected in the graph: many other singers points to these three singers.

\section{Conclusion and Future Work}

In this paper, we have proposed a comment-based video categorization approach by using NMF. Experimental results showed that our comment-based clustering yields better performance than that of tag-based approach which was proposed previously in the literature. On the other hand, we have successfully discovered some non-trivial categories among the videos of Hong Kong singers. Since our categorization is learnt from user feedbacks, it can provide an easy way for users to reach their desire videos via our list of categories. Additionally, our comment-based clustering can reveal relationships among singers within the social network which can help in the context of social culture study.

In our future work, we plan to extend the comment-based technique from video and singer clustering to user clustering. After we have obtained the three types of cluster, we can acquire the relationships among different videos, singers and users by analyzing the inter-cluster similarity. As such, social culture can be studied by combining and analyzing the discovered relationships. With the video-video, singer-singer, user-singer, and user-user relationships found by clustering, we can know the changes in music styles and singer styles over the ages, the trend of music, the ways people appreciate music, and even the special relationships of singers reflected by news, and more. Relationships observed by clustering are not only useful for social scientists to study social culture, but also beneficial for businesses, entertainment companies, fans clubs, social network systems and system users. With the help of examined useruser relationships, businesses can be profited from reducing advertising costs by advertise only to the potential customer groups. User-signer relationships define user-idol groups, entertainment companies can effectively promote to the target groups. Determining the user-singer relationships, in addition to profits for entertainment companies, fans groups can easily be managed. Other than the advantages for some specific parties, general users are also benefited. Well-clustered groups of videos and singers equipped with a batch of indicative words leads to a effort saving video searching for users. Also, social network systems are able to detect and refine incorrect tags with the indicative words resulted from clustering. As a result, introductions to videos are more precise and thus improves the video searching function. 


\section{References}

[1] X. Cheng, C. Dale, and J. Liu. Understanding the characteristics of internet short video sharing: Youtube as a case study. In CoRR abs, Jul 2007.

[2] L. Guo, S. Jiang, L. Xiao, and X. Zhang. Fast and lowcost search schemes by exploiting localities in $\mathrm{p} 2 \mathrm{p}$ networks. J. Parallel Distrib. Comput., 65(6):729742,2005

[3] J. Heer and D. Boyd. Vizster: Visualizing online social networks. IEEE Symposium on Information Visualization, 2005.

[4] C. M. C. Y. Julia Stoyanovich, Sihem AmerYahia. Leveraging tagging to model user interests in del.icio.us. In $A A A I$ '08: Proceedings of the 2008 AAAI Social Information Spring Symposium. AAAI, 2008.

[5] P. P. Kotsiantis S., Kanellopoulos D. Multimedia mining. In WSEAS Transactions on Systems, Issue 10, Volume 3, pages 3263-3268, December 2004.

[6] X. Li, L. Guo, and Y. E. Zhao. Tag-based social interest discovery. In WWW '08: Proceeding of the 17th international conference on World Wide Web, pages 675-684, New York, NY, USA, 2008. ACM.

[7] C. G. R. A. A. F. L. Rodrygo L. T. Santos, Bruno P. S. Rocha. Characterizing the youtube video-sharing community. 2007.

[8] M. F. Schwartz and D. C. M. Wood. Discovering shared interests using graph analysis. Commun. ACM, 36(8):78-89, 1993.

[9] K. Sripanidkulchai, B. Maggs, and H. Zhang. Efficient content location using interest-based locality in peer-to-peer systems. In INFOCOM 2003. TwentySecond Annual Joint Conference of the IEEE Computer and Communications Societies. IEEE, volume 3, pages 2166-2176 vol.3, 2003.

[10] W. Xu, X. Liu, and Y. Gong. Document clustering based on non-negative matrix factorization. In SIGIR '03: Proceedings of the 26th annual international ACM SIGIR conference on Research and development in informaion retrieval, pages 267-273, New York, NY, USA, 2003. ACM.

[11] L. Yang, J. Liu, X. Yang, and X.-S. Hua. Multimodality web video categorization. In MIR '07: Proceedings of the international workshop on Workshop on multimedia information retrieval, pages 265-274, New York, NY, USA, 2007. ACM.
[12] O. R. Zaïane, J. Han, Z.-N. Li, S. H. Chee, and J. Y. Chiang. Multimediaminer: a system prototype for multimedia data mining. In SIGMOD '98: Proceedings of the 1998 ACM SIGMOD international conference on Management of data, pages 581-583, New York, NY, USA, 1998. ACM. 